\begin{tabular}{|c|l|}
\hline Title & $\begin{array}{l}\text { Mechanisms of trichloramine removal with activated carbon: Stoichiometric analysis with isotopically labeled } \\
\text { trichloramine and theoretical analysis with a diffusion-reaction model }\end{array}$ \\
\hline Author(s) & Sakuma, Miki; Matsushita, Taku; Matsui, Y oshihiko; A ki, Tomoko; Isaka, Masahito; Shirasaki, Nobutaka \\
\hline Citation & $\begin{array}{l}\text { Water research, 68, 839-848 } \\
\text { https://doi.org/10.1016/.watres.2014.10.051 }\end{array}$ \\
\hline Issue Date & 2015-01-01 \\
\hline Doc URL & http://hdl.handle.net/2115/58003 \\
\hline Type & article (author version) \\
\hline File Information & matsushita_150303.pdf \\
\hline
\end{tabular}

Instructions for use 


\title{
Mechanisms of trichloramine removal with activated carbon: Stoichiometric analysis with isotopically labelled trichloramine and theoretical analysis with a diffusion-reaction model
}

\author{
Miki Sakuma, Taku Matsushita*, Yoshihiko Matsui, Tomoko Aki, Masahito Isaka, Nobutaka Shirasaki \\ Graduate School of Engineering, Hokkaido University, N13W8, Sapporo 060-8628, Japan
}

* Corresponding author. e-mail, taku-m@eng.hokudai.ac.jp; Tel./fax: +81-11-706-7279

\begin{abstract}
This study investigated the mechanism by which activated carbon removes trichloramine, a byproduct of water treatment that has a strongly offensive chlorinous odor. A stoichiometrical mass balance for ${ }^{15} \mathrm{~N}$ before and after activated carbon treatment of laboratory-prepared ${ }^{15} \mathrm{~N}$-labelled trichloramine solutions clearly revealed that the mechanism of trichloramine removal with activated carbon was not adsorption but rather reductive decomposition to nitrogen gas. There was a weak positive correlation between the surface decomposition rate constant of trichloramine and the concentration of basic functional groups on the surface of the carbon particles, the suggestion being that the trichloramine may have been reduced by sulfhydryl groups $(-\mathrm{SH})$ on the activated carbon surface. Efficient decomposition of trichloramine was achieved with super powdered activated carbon (SPAC), which was prepared by pulverization of commercially available PAC into very fine particles less than $1 \mu \mathrm{m}$ in diameter. SPAC could decompose trichloramine selectively, even when trichloramine and free chlorine were present simultaneously in water, the indication being that the strong disinfection capability of residual free chlorine could be retained even after trichloramine was effectively decomposed. The residual ratio of trichloramine after carbon contact increased somewhat at low water temperatures of $1-5{ }^{\circ} \mathrm{C}$. At these low temperatures, biological treatment, the traditional method for control of a major trichloramine precursor (ammonium nitrogen), is inefficient. Even at these low temperatures, SPAC could reduce the trichloramine concentration to an acceptable level. A theoretical analysis with a diffusion-reaction model developed in the present study revealed that the increase in the trichloramine residual with decreasing water temperature was attributable to the temperature dependence of the rate of the reductive reaction rather than to the temperature dependence of the diffusive mass transfer rate.
\end{abstract}

Keywords: chlorinous odor; trichloramine; super powder activated carbon; drinking water treatment

\section{Nomenclature}

$C_{\mathrm{I}}(t, R) \quad$ liquid phase concentration on the outer surface of an adsorbent particle $(\mu \mathrm{g} / \mathrm{L})$

$C(t) \quad$ adsorbate concentration in bulk water phase as a function of time, $t(\mu \mathrm{g} / \mathrm{L})$ 


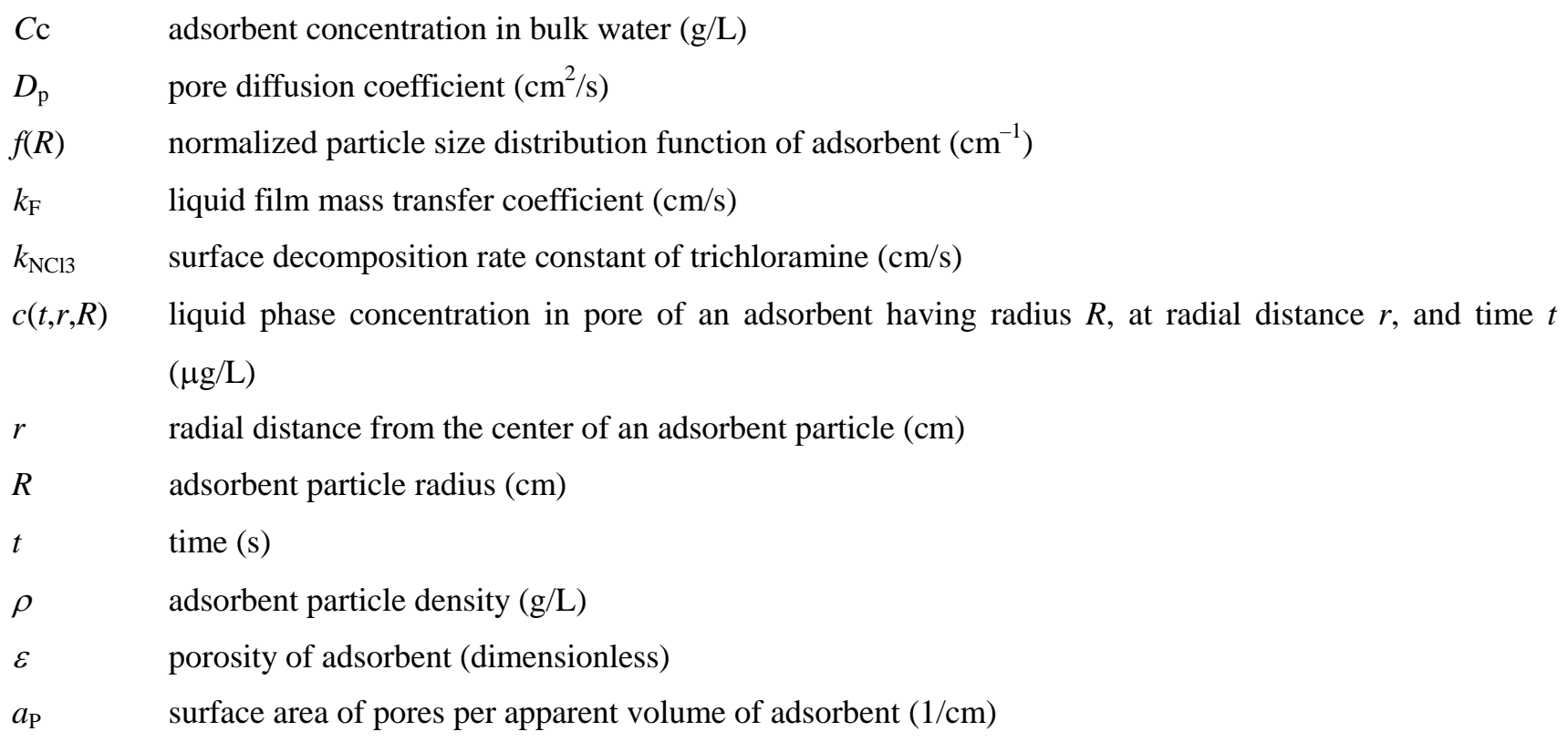

\section{Introduction}

Handling customers' complaints and dissatisfaction regarding the taste and odor of tap water are important issues that practitioners in drinking water treatment plants sometimes face. Chlorinous odor is the leading cause of dissatisfaction among persons who drink water in regions where chlorination or chloramination is employed for disinfection of drinking water (Piriou et al. 2004). Trichloramine $\left(\mathrm{NCl}_{3}\right)$ and dichloramine $\left(\mathrm{NHCl}_{2}\right)$ are recognized as the compounds that cause the chlorinous odor in tap water; trichloramine causes a much stronger chlorinous odor than dichloramine (Kranser and Barret 1984). Trichloramine is formed mainly by the reaction between ammonium ions, which are present in raw sources of drinking water, and the chlorine used for disinfection. The removal of ammonium ions before the chlorination process is one strategy for mitigating the problem of trichloramine formation. Water treatment involving slow sand filtration (Štembal et al. 2005) or activated carbon (Andersson et al. 2001) are examples of biological methods used to remove ammonium ions. However, the activity of nitrifying microorganisms is reduced by low water temperatures, and in cold regions or during the winter nitrification becomes small to remove ammonium ions efficiently. To solve the trichloramine formation problem when the water temperature is low, treatment technologies that are independent of biological activity are required.

Treatment with activated carbon is the traditional method used to remove dichloramine and monochloramine $\left(\mathrm{NH}_{2} \mathrm{Cl}\right)$ in water treatment (Bauer and Snoeyink 1973, Snoeyink and Suidan 1975). However, sufficient removal requires a very long contact time with the carbon. For example, almost $2 \mathrm{~d}$ (45 h) is required to remove dichloramine by using powdered activated carbon (PAC) with diameters of 149-177 $\mu$ m (Bauer and Snoeyink 1973, Snoeyink and Suidan 1975). Therefore, although PAC treatment is a simple process, its slow removal rate detracts from its application as a technology for drinking water treatment. Decreasing the size of the activated carbon particles generally enhances the removal rate achieved with activated carbon, because size reduction increases the specific surface area (surface area per unit weight of activated carbon). However, the technology of pulverizing 
activated carbon particles has typically produced particles no smaller than $5 \mu \mathrm{m}$ in diameter (Matsui et al. 2008). Recently, our research group developed a way to produce super fine activated carbon particles (SPAC) with diameters less than $1 \mu \mathrm{m}$ by pulverizing conventionally sized PAC. We have reported highly improved adsorptive uptake rates of natural organic matter (NOM) (Matsui et al. 2005) and of compounds that produce earthy-musty odors (Matsui et al. 2007, Matsui et al. 2009). We hypothesized that treatment with SPAC may also be an effective way to remove trichloramine efficiently. A preliminarily study conducted by our research group actually found that SPAC was superior to PAC for removal of trichloramine and dichloramine (Matsui et al. 2008). However, sufficient studies have not been conducted on the effects of water temperature and SPAC characteristics on trichloramine and dichloramine removal.

In general, an important characteristic of activated carbon is its high adsorption affinity and high capacity to adsorb various target compounds to be removed from raw drinking water sources. Activated carbon has therefore been widely used in drinking water treatment plants for a long time to reduce contaminant levels of NOM (Çapar and Yetış 2002), earthy-musty odor compounds (Herzing et al. 1977), and pesticides (Robeck et al. 1965). In contrast to the adsorptive removal of these compounds, the removal of dichloramine and monochloramine by activated carbon has been reported to be due to reductive decomposition of these compounds on the surface of the carbon (Bauer and Snoeyink 1973, Snoeyink and Suidan 1975). It has been speculated that dichloramine reacts with functional groups located on the internal surfaces of activated carbon particles and is then reduced to nitrogen gas (Bauer and Snoeyink 1973, Snoeyink and Suidan 1975). However, no direct evidence for the reductive decomposition of dichloramine has been presented so far. The mechanism of trichloramine removal by activated carbon is also unclear.

The objectives of this study were 1) to provide direct evidence of the trichloramine removal mechanism involving reductive decomposition of trichloramine to nitrogen gas by activated carbon and 2) to model the effect of water temperature on trichloramine removal. The removal mechanism was investigated by 1) comparing the masses of ${ }^{15} \mathrm{~N}$ before and after treatment of laboratory-prepared, ${ }^{15} \mathrm{~N}$-labelled trichloramine solutions, 2) comparing the trichloramine removal performances of activated carbon substrates with various surface characteristics, and 3) modeling the process of trichloramine removal at different temperatures in a diffusion-reaction system. Simulating the effect of water temperature enabled us to judge whether SPAC treatment could be a practical way to control chlorinous odors at low water temperatures.

\section{Materials and Methods}

\subsection{Activated carbon}

Seven commercially available PACs (four wood-based, two coconut-shell-based, and one coal-based PAC) were obtained from manufacturers (Table 1). These were pulverized to superfine particles (SPAC) with a wet bead mill (Metawater Co., Tokyo, Japan). The carbons were stored as slurries at $4{ }^{\circ} \mathrm{C}$ prior to use. 
Table 1 Characteristics of SPACs used.

\begin{tabular}{|c|c|c|c|c|c|c|c|c|c|c|c|c|c|c|c|c|}
\hline \multirow{2}{*}{ Name } & \multirow{2}{*}{$\begin{array}{c}\text { Raw } \\
\text { material }\end{array}$} & \multirow{2}{*}{$\begin{array}{l}\mathrm{D}_{50} \\
\mu \mathrm{m} \\
\end{array}$} & \multicolumn{3}{|c|}{ Pore surface area, $\mathrm{m}^{2} / \mathrm{g}$} & \multicolumn{2}{|c|}{ Pore volume, mL/g } & \multicolumn{2}{|c|}{ Functional group, $\mu \mathrm{eq} / \mathrm{g}$} & \multicolumn{4}{|c|}{ Elemental composition, \% } & \multirow{2}{*}{$\begin{array}{l}\mathrm{ZP} \\
\mathrm{mV}\end{array}$} & \multirow{2}{*}{ IEP } & \multirow{2}{*}{ pHpzc } \\
\hline & & & BET & micro & meso & micro & meso & Basic & Acidic & $\mathrm{C}$ & $\mathrm{N}$ & $\mathrm{S}$ & $\mathrm{O}$ & & & \\
\hline wood-1 & wood & 0.53 & 1174 & 918 & 88 & 0.405 & $\begin{array}{r}0.136 \\
\end{array}$ & 780 & 351 & 81.9 & 0.2 & 0.2 & 8.2 & -46 & 2.8 & 7.4 \\
\hline wood-2 & wood & 0.47 & 1111 & 953 & 131 & 0.450 & 0.252 & 711 & 193 & 84.6 & 0.2 & 0.1 & 9.1 & -39 & 3.3 & 8.7 \\
\hline wood-3 & wood & 0.52 & 1145 & 919 & 138 & 0.395 & 0.223 & 790 & 350 & 85.3 & 0.1 & 0.1 & 8.9 & -42 & 4.2 & 8.9 \\
\hline wood-4 & wood & 0.62 & 1130 & 922 & 116 & 0.388 & 0.392 & 696 & 553 & 83.3 & 0.2 & 0.1 & 8.9 & -46 & 2.9 & 7.7 \\
\hline coco-1 & coconut shell & 0.57 & 978 & 824 & 103 & 0.302 & 0.310 & 329 & 425 & 88.1 & 0.2 & 0.1 & 6.1 & -42 & 3.9 & 6.4 \\
\hline coco-2 & coconut shell & 0.50 & 1215 & 1023 & 79 & 0.432 & 0.133 & 582 & 433 & 89.1 & 0.2 & 0.1 & 6.1 & -48 & 3.3 & 7.3 \\
\hline coal & coal & 0.49 & 884 & 637 & 55 & 0.281 & 0.314 & 366 & 757 & 79.2 & 0.4 & 0.6 & 11.0 & -38 & 4.2 & 6.3 \\
\hline
\end{tabular}

$\mathrm{D}_{50}$, median diameter; micro, micropore; meso, mesopores; $\mathrm{ZP}$, zeta potential; IEP, isoelectric point; $\mathrm{pHpzc}$, pH at point of zero charge.

\subsection{Preparation of trichloramine and ${ }^{15} \mathrm{~N}$-labelled trichloramine solutions}

Trichloramine was prepared according to the method reported by Kosaka et al. (2010). Phosphate buffer (pH 7.0, 5 mM sodium phosphate) was prepared with Milli-Q water (Milli-Q Advantage, Millipore Co., Bedford, MA, USA) and then supplemented with $0.25 \mathrm{mg}-\mathrm{N} / \mathrm{L}$ ammonium chloride at room temperature. The solution was further supplemented with $4 \mathrm{mg}-\mathrm{Cl}_{2} / \mathrm{L}$ of sodium hypochlorite and mixed at $400 \mathrm{rpm}$ for 30 min with a magnetic stirrer. The solution was allowed to stand for $1.5 \mathrm{~h}$ at a room temperature of about $20^{\circ} \mathrm{C}$ and then stored overnight at $4{ }^{\circ} \mathrm{C}$ in the dark. The resulting solution contained approximately $0.2-0.4$ and $1.5-2.0 \mathrm{mg}-\mathrm{Cl}_{2} / \mathrm{L}$ of trichloramine and residual free chlorine, respectively, the trichloramine concentrations being one order larger than those reported in tap waters $\left(<0.015-0.046 \mathrm{mg}-\mathrm{Cl}_{2} / \mathrm{L}\right)\left(\right.$ Kosaka et al. 2010). A ${ }^{15} \mathrm{~N}$-labelled trichloramine solution was prepared in the same manner as described above with a ${ }^{15} \mathrm{~N}$-labelled ammonium chloride solution containing $0.3 \mathrm{mg}-\mathrm{N} / \mathrm{L}$ and a sodium hypochlorite solution containing $4.8 \mathrm{mg}-\mathrm{Cl}_{2} / \mathrm{L}$. In addition to the ${ }^{15} \mathrm{~N}$-labelled trichloramine, the solution contained dissolved ${ }^{15} \mathrm{~N}_{2}$ gas that had been generated during the chlorination of ${ }^{15} \mathrm{~N}$-labelled ammonium chloride (Bauer and Snoeyink 1973).

\section{$2.3{ }^{15} \mathrm{~N}$ mass balance experiments}

The laboratory-prepared, ${ }^{15} \mathrm{~N}$-labelled trichloramine solution, which contained ${ }^{15} \mathrm{~N}_{2}$ as well as ${ }^{15} \mathrm{~N}$-labelled trichloramine, was transferred to the lower chamber of a glass vessel with separable upper and lower chambers. The lower chamber was tightly sealed with a separator made of aluminum foil (Fig. 1A). The gas in the upper chamber of the vessel was replaced with Ar gas. To purge ${ }^{15} \mathrm{~N}_{2}$ dissolved in the solution into the gas phase, the solution was intensely mixed for 20 min with the gas phase after the separator was removed and was then kept in a stationary mode for $30 \mathrm{~min}$ (Fig. 1B). An aliquot of the gas was withdrawn from the upper chamber to measure the ${ }^{15} \mathrm{~N}_{2}$ concentration (Fig. 1C). The same steps corresponding to Figure $1 \mathrm{~A}-\mathrm{C}$ were repeated (Fig. 1D-F) to purge ${ }^{15} \mathrm{~N}_{2}$ completely from the solution. An aliquot of the solution was withdrawn from the lower chamber (Fig. 1G) to measure the initial concentrations of chloramines, and then an aliquot of phosphate buffer with the same volume as the aliquot that had been withdrawn was added to the solution to ensure that there was no head space between the surface of the solution and the separator. Trichloramine was quantified by headspace gas chromatography/mass spectrometry (HS-GC/MS) as described below. The solution was supplemented with $3 \mathrm{mg} / \mathrm{L}$ of SPAC (wood-1) (Fig. 1H), tightly sealed with the separator, and then mixed for 10 min with a magnetic stirrer (Fig. 1I). During the 
mixing, the gas in the upper chamber was replaced with Ar gas (Fig. 1J). An aliquot of the gas in the chamber was withdrawn to measure the initial concentration of ${ }^{15} \mathrm{~N}_{2}$. After $10 \mathrm{~min}$ of mixing, the separator was removed. The liquid phase was intensely mixed with the gas phase for $20 \mathrm{~min}$ and then left in a stationary mode for $30 \mathrm{~min}$ to allow any ${ }^{15} \mathrm{~N}_{2}$ gas generated by the reaction between trichloramine and the SPAC to transfer to the gas phase from the solution (Fig. 1K). An aliquot of the gas phase was withdrawn from the upper chamber to measure the ${ }^{15} \mathrm{~N}_{2}$ concentrations (Fig. 1L). To measure the chloramine concentrations, an aliquot of the solution was withdrawn (Fig. $1 \mathrm{M}$ ) and then filtered through a membrane filter ( $\phi=0.2 \mu \mathrm{m}$, PTFE, Toyo Roshi Kaisha, Ltd., Tokyo, Japan) to remove any SPAC particles. This experiment was conducted at $20^{\circ} \mathrm{C}$ three times: these replicates are designated as Experiments 1, 2, and 3.

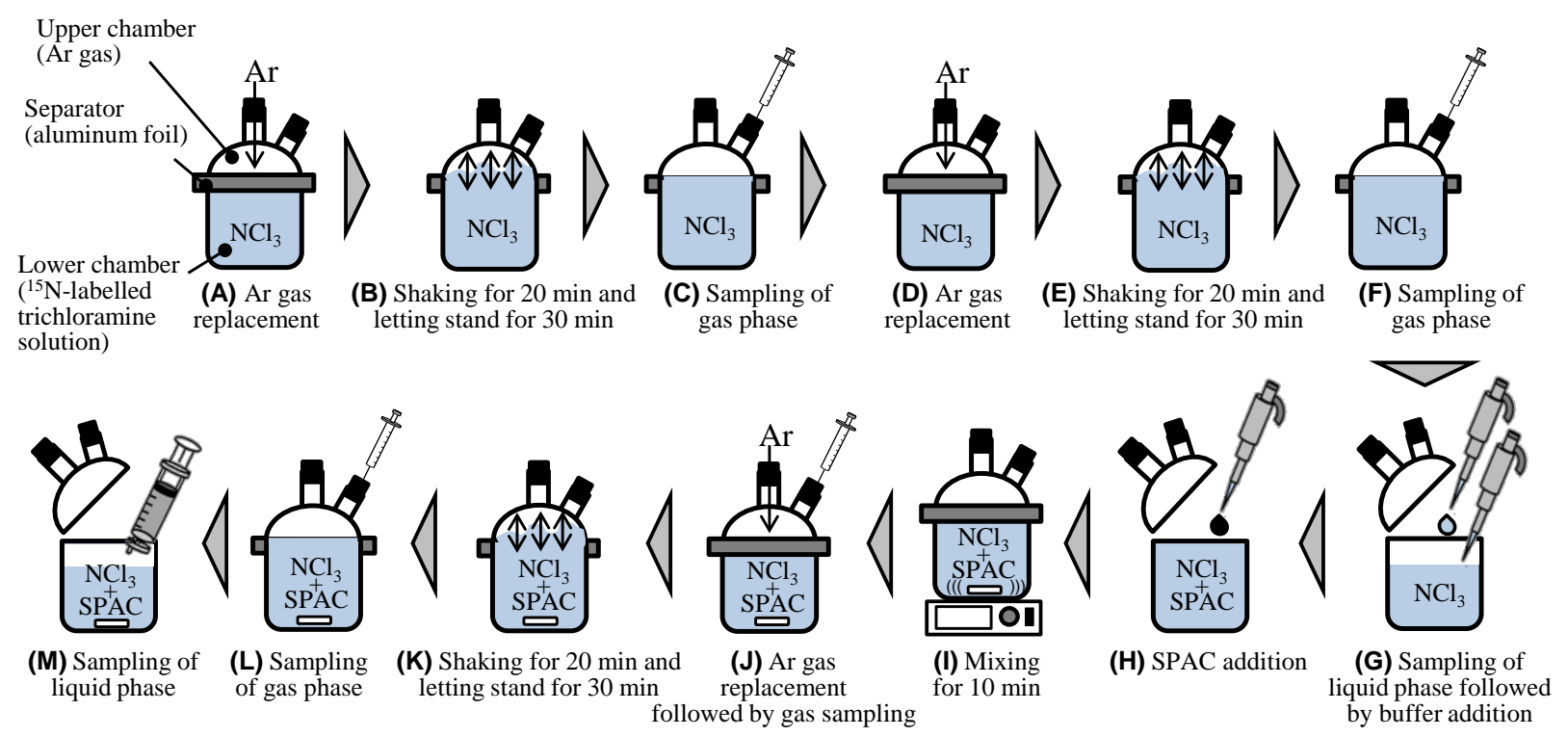

Fig. 1 Protocol for ${ }^{15} \mathrm{~N}$ mass balance experiments.

\subsection{Batch decomposition of trichloramine with SPACs}

The trichloramine solution was transferred to a one-liter glass beaker. The solution was supplemented with PAC at a concentration of $1 \mathrm{mg} / \mathrm{L}$ and then covered with a floating lid. The lid, made of a polystyrene form covered with aluminum foil, prevented volatilization of the chloramines. The solution was stirred with a magnetic stirrer, and samples were withdrawn from the beaker though a siphon at predetermined time intervals. The concentrations of chloramines and free chlorine were measured with the $N, N$-diethyl-p-phenylenediamine (DPD) colorimetric method (American Public Health Association/American Water Works Association/Water Environment Federation 2005) after the sample had been filtered through a membrane filter ( $\phi=0.2 \mu \mathrm{m}$, PTFE, Toyo Roshi Kaisha, Ltd.). The experiments were conducted at water temperatures of 1,5 , or $20^{\circ} \mathrm{C}$.

\subsection{Quantifications of trichloramine, ${ }^{15} \mathrm{~N}$-labelled trichloramine, other chloramines, and ${ }^{15} \mathrm{~N}_{2}$ gas}

The HS-GC/MS analysis of trichloramine (Kosaka et al. 2010) was carried out as follows: separation was 
performed using an Agilent 7890A gas chromatograph (Agilent Technologies, Inc., CA, USA) equipped with an HP-1MS capillary column ( $30 \mathrm{~m} \times 0.25 \mathrm{~mm}, 0.25 \mu \mathrm{m}$; Agilent Technologies). The injection temperature was $40{ }^{\circ} \mathrm{C}$, and the flow rate of He was $2.0 \mathrm{~mL} / \mathrm{min}$. The injection mode was pulsed-split, and the split ratio was 1:30. The oven temperature was constant at $30^{\circ} \mathrm{C}$. Detection was performed using an Agilent 5975C mass spectrometer (Agilent Technologies) in selected ion monitoring (SIM) mode. The auxiliary temperature, quadrupole temperature, and ion source temperature were controlled at 110,150 , and $150{ }^{\circ} \mathrm{C}$, respectively. The detected fragment ions of trichloramine, ${ }^{15} \mathrm{~N}$-labelled trichloramine, and $\mathrm{d}_{3}$-labelled 1,1,2-trichloroethane (internal standard) occurred at $\mathrm{m} / \mathrm{z}$ of 84,85 , and 100, respectively. Dichloramine, monochloramine, and free chlorine were measured according to the DPD colorimetric method.

${ }^{15} \mathrm{~N}_{2}$ gas was quantified with a GC/MS equipped with a CP-Molsieve $5 \AA$ capillary column $(50 \mathrm{~m} \times 320 \mu \mathrm{m} \times$ $30 \mu \mathrm{m}$; Agilent Technologies). The temperatures of the ion source, injector, and transfer line were controlled at 230, 200 and $270{ }^{\circ} \mathrm{C}$, respectively. The GC/MS was operated in the selected ion monitoring (SIM) mode with ${ }^{38} \mathrm{Ar}$ as an internal standard; the detected fragment ions of ${ }^{15} \mathrm{~N}_{2}$ and ${ }^{38} \mathrm{Ar}$ occurred at $\mathrm{m} / \mathrm{z}$ of 30 and 38 , respectively.

\subsection{Measurement of physicochemical properties of SPACs}

Particle size distributions of activated carbons were determined with a laser diffraction and scattering instrument (Microtrac MT3000 II, Nikkiso Co., Ltd., Tokyo, Japan). The median particle diameter $\left(\mathrm{D}_{50}\right)$ was calculated from the particle size distribution.

Specific surface areas and pore size distributions of the SPACs were analyzed by nitrogen gas adsorption at 77 K with an automated gas sorption analyzer (Autosorb-iQ-MP; Quantachrome Instruments, Boynton Beach, FL, USA). Specific surface area $\left(\mathrm{S}_{\mathrm{BET}}\right.$ ) was determined by using the BET (Brunauer-Emmett-Teller) method. Pore size distributions were determined by a combination of two widely accepted models: the DFT (Density Functional Theory) model for the pore size distribution of micropores $(<2 \mathrm{~nm})$ and the BJH (Barrett-Joyner -Halenda) model for mesopores and macropores $(>2 \mathrm{~nm})$. Elemental analysis of the SPACs was carried out with an elemental analyzer (Vario EL III, Elemental Analysensysteme GmbH, Hanau, Germany).

Surface acidity and basicity of the SPACs were determined by using the Boehm titration method (Boehm 1994, 2002) with some modifications. SPACs were dried at $105^{\circ} \mathrm{C}$ for $12 \mathrm{~h}$ in a drying oven. After cooling, an aliquot of $200 \mathrm{mg}$ of the SPAC was transferred to a test tube, and $10 \mathrm{~mL}$ of $0.05 \mathrm{M}$ sodium hydroxide $(\mathrm{NaOH})$ or hydrogen chloride $(\mathrm{HCl})$ was added. The suspension was placed under vacuum for $1 \mathrm{~h}$ to remove any air from the pores and then vortexed intensely for $24 \mathrm{~h}$ to allow the surface functional groups of the SPAC to react with the base or acid. After vortexing, the suspension was filtered through a membrane filter to remove any SPAC particles. Aliquots (5 $\mathrm{mL}$ ) of the filtrate were transferred to beakers, and then an aliquot of $15 \mathrm{~mL}$ of $0.05 \mathrm{M} \mathrm{HCl}$ or $\mathrm{NaOH}$ was added to each filtrate of the $\mathrm{NaOH}$-treated or HCl-treated SPACs, respectively. The acidic or basic filtrates were neutralized by back titrating with $\mathrm{NaOH}$ or $\mathrm{HCl}$, respectively.

The zeta potential of the SPACs was measured with a dynamic, light-scattering, laser zeta potential analyzer (Zetasizer Nano ZS, Malvern Instruments Ltd., Malvern, UK) in the same phosphate buffer used for the preparation of the trichloramine solutions. The isoelectric point (IEP) was determined by measuring the zeta potential of the 
SPACs as the $\mathrm{pH}$ of the solution was changed.

The $\mathrm{pH}$ at the point of zero charge $\left(\mathrm{pH}_{\mathrm{pzc}}\right)$ indicates the condition of the surface of the activated carbon when it has no net charge in solution. The initial $\mathrm{pH}$ was adjusted to the range $2-8$ by adding $0.1 \mathrm{M} \mathrm{HCl}$ or $\mathrm{NaOH}$ to $50 \mathrm{~mL}$ of $0.01 \mathrm{M}$ sodium chloride $(\mathrm{NaCl})$. An aliquot of $150 \mathrm{mg}$ of SPAC was added to each of the different $\mathrm{pH}$ solutions, and the solutions were agitated for $48 \mathrm{~h}$. The equilibrium $\mathrm{pH}$ was plotted against the initial $\mathrm{pH}$. The $\mathrm{pH}_{\mathrm{pzc}}$ was the initial $\mathrm{pH}$ that was identical to the equilibrium $\mathrm{pH}$ (Noh and Schwarz 1990).

\subsection{Theoretical analysis}

When the size distribution of adsorbent particles is taken into consideration, the mass balance for an adsorbate in a batch container is described by the following equation:

$$
\frac{\mathrm{d} C(t)}{\mathrm{d} t}=-\frac{3 C_{\mathrm{c}} k_{\mathrm{F}}}{\rho} \int_{0}^{\infty} \frac{f(R)}{R}\left[C(t)-C_{\mathrm{I}}(t, R)\right] \mathrm{d} R
$$

For highly adsorbent substrates, intraparticle migration of the adsorbate is mainly the result of surface diffusion, because most of the adsorbate particles adsorb onto the surface of the adsorbents, and the fraction of the adsorbate in the liquid phase of the pores is negligibly small. The large $K_{\text {ow }}$ of trichloramine $(-1.6$ calculated with Estimation Programs Interface Suite software, United States Environmental Protection Agency), however, suggests that it is very unlikely to adsorb to activated carbon. Surface diffusion is therefore not expected to account for most intraparticle migration of trichloramine. Instead, radial mass transport of trichloramine molecules occurs in the liquid phase within the pores by pore diffusion. We have assumed that the trichloramine decomposition that occurs on the surface of pores in carbon particles follows first-order reaction kinetics. Finally, the rate of change of the liquid phase concentration of trichloramine in pores is described by the following equation:

$$
\varepsilon \frac{\partial c(t, r, R)}{\partial t}=\frac{1}{r^{2}} \frac{\partial}{\partial r}\left\{D_{\mathrm{P}} r^{2}\left[\frac{\partial c(t, r, R)}{\partial r}\right]\right\}-\varepsilon \cdot a_{\mathrm{P}} k_{\mathrm{NCl}_{3}} \cdot c(t, r, R)
$$

The mass balance of trichloramine with respect to a carbon particle is described by equating the rate of change of the amount of trichloramine in a carbon particle, the rate of mass transfer from the external particle surface to the inside of the particle, and the rate of decomposition of trichloramine as follows:

$$
\varepsilon \frac{\mathrm{d}}{\mathrm{d} t}\left\{\int_{0}^{R} c(t, r, R) \cdot 4 \pi r^{2} \mathrm{~d} r\right\}=k_{\mathrm{F}}\left[C(t)-c_{\mathrm{I}, i}(t)\right] \cdot 4 \pi R^{2}-\varepsilon \cdot a_{\mathrm{P}} k_{\mathrm{NCl}_{3}} \int_{0}^{R} c(t, r, R) \cdot 4 \pi r^{2} \mathrm{~d} r
$$

The particle size distribution was approximated by a discrete density function. The set of model equations was converted into a set of ordinary differential equations with respect to time $t$ using the method of orthogonal collocation. The resulting equations were solved as a system of ordinary differential equations by the Gear's stiff 
method in the International Mathematics and Statistics Library (IMSL, Visual Numerics, Houston, TX, USA), after deriving the analytical Jacobian of the equations. Details are presented in Supplementary content.

A single pore diffusion coefficient $\left(D_{\mathrm{p}}\right)$ at a given water temperature was used, irrespective of carbon particle size. Mass transfer across the liquid film external to the adsorbent particle surfaces cannot be the rate-determining step in well mixed reactors (Sontheimer et al. 1988), and resistance to such mass transfer was therefore neglected. For purposes of modeling mass transfer in the model simulations, the liquid film mass transfer coefficient $\left(k_{\mathrm{F}}\right)$ was set to $0.1 \mathrm{~cm} / \mathrm{s}$. Coefficients larger than this value produced the same simulated trichloramine concentrations, the indication being that the $k_{\mathrm{F}}$ value did not limit the rate of decomposition of trichloramine by PAC.

\section{Results and discussion}

\section{$3.1{ }^{15} \mathrm{~N}$ mass balance experiments}

After the ${ }^{15} \mathrm{~N}$-labelled trichloramine solution was intensely mixed with the gas phase in order to purge the ${ }^{15} \mathrm{~N}_{2}$ gas that had been produced during the preparation of the ${ }^{15} \mathrm{~N}$-labelled trichloramine solution by the reaction of ${ }^{15} \mathrm{~N}$-labelled ammonium ions with chlorine, ${ }^{15} \mathrm{~N}_{2}$ was detected in the gas phase (Fig. 1C) at 110-192 ppm. However, no ${ }^{15} \mathrm{~N}_{2}$ gas was detected $(<1.4 \mathrm{ppm})$ in the gas phase (Fig. $\left.1 \mathrm{~F}\right)$ after the solution was again intensely mixed with the gas phase. Therefore ${ }^{15} \mathrm{~N}_{2}$ was almost completely removed from the ${ }^{15} \mathrm{~N}$-labelled trichloramine solution. Even if a small fraction of the ${ }^{15} \mathrm{~N}_{2}$ remained in solution, the ${ }^{15} \mathrm{~N}_{2}$ was not potentially transferrable to the gas phase. The ${ }^{15} \mathrm{~N}$-labelled trichloramine solutions contained 232, 243, and $370 \mu \mathrm{g}-\mathrm{Cl}_{2} / \mathrm{L}$ of trichloramine in Experiments 1-3, respectively. Neither dichloramine nor monochloramine was detected, the indication being that the ${ }^{15} \mathrm{~N}$-labelled trichloramine solution contained only trichloramine as a sole source of nitrogen before the reaction with the SPAC. The amount of ${ }^{15} \mathrm{~N}$ from the ${ }^{15} \mathrm{~N}$-labelled trichloramine solution $(600 \mathrm{~mL}$ ) was calculated to be $10.3,10.3$, and 15.7 $\mu g-{ }^{15} \mathrm{~N}$ in Experiments 1-3, respectively (white vertical bars in Fig. 2).

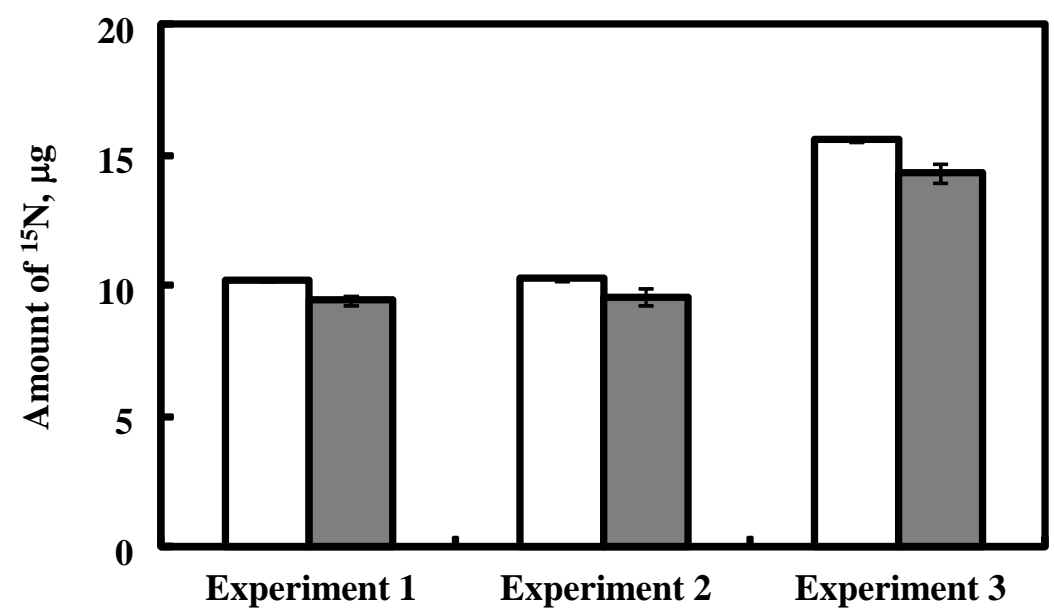

Fig. 2 Comparison of amount of ${ }^{15} \mathrm{~N}$ before and after SPAC treatment (wood-1). White and gray bars indicate the amount of ${ }^{15} \mathrm{~N}$ before (liquid phase, as trichloramine) and after (gas phase, as $\mathrm{N}_{2}$ gas) the SPAC treatment, respectively. Error bars indicate standard deviations of three measurements. 
After addition of the SPAC and mixing of the trichloramine solution, none of chloramines was detected in the solution (Fig. 1M), the indication being that the ${ }^{15} \mathrm{~N}$-labelled trichloramine was completely removed by the SPAC. In contrast, ${ }^{15} \mathrm{~N}_{2}$ gas was detected in the gas above the solution, which had been in contact with the solution (Fig. 1L). The ${ }^{15} \mathrm{~N}_{2}$ concentrations in the gas phase were 51, 52, and $78 \mathrm{ppm}$, equivalent to 9.5, 9.6, and $14.4 \mu \mathrm{g}-{ }^{15} \mathrm{~N}$, respectively (the volume of the gas phase was $150 \mathrm{~mL}$ ), as shown in Figure 2 (gray bars). Before addition of the SPAC, the amount of ${ }^{15} \mathrm{~N}_{2}$ that was potentially transferrable to the gas phase from the ${ }^{15} \mathrm{~N}$-labelled trichloramine solution was undetectably small, as already noted. The solution therefore contained only trichloramine as a source of nitrogen. The ${ }^{15} \mathrm{~N}_{2}$ in the gas phase must therefore have originated from the ${ }^{15} \mathrm{~N}$-labelled trichloramine that had been removed by the SPAC. Moreover, the amounts of nitrogen detected in the gas phase were almost the same as those previously present in the ${ }^{15} \mathrm{~N}$-labelled trichloramine solutions. This result clearly demonstrates that trichloramine was reductively decomposed to nitrogen gas by activated carbon treatment.

It is well known that activated carbon can reductively decompose free chlorine to chloride ions (Giles and Danell 1983, Snoeyink and Suidan 1972, Suidan et al. 1977). Activated carbon has also been reported to reductively decompose bromate (Bao et al. 1999, Siddiqui et al. 1996) and chlorite (Gonce and Voudrias 1994, Voudrias et al. 1983). Snoeyink and his research group (Bauer and Snoeyink 1973, Snoeyink and Suidan 1975) have stated that dichloramine is reduced to nitrogen gas by contact with activated carbon as follows:

$$
2 \mathrm{NHCl}_{2}+\mathrm{C}^{*}+\mathrm{H}_{2} \mathrm{O} \rightarrow \mathrm{N}_{2}+\mathrm{C} * \mathrm{O}+4 \mathrm{H}^{+}+4 \mathrm{Cl}^{-}
$$

where $\mathrm{C}^{*}$ and $\mathrm{C} * \mathrm{O}$ represent a reductive functional group on the surface of an activated carbon particle and a surface oxide on the carbon surface, respectively. Likewise, we hypothesized the following reaction for the reductive decomposition of trichloramine to nitrogen gas:

$$
2 \mathrm{NCl}_{3}+3 \mathrm{C}^{*}+3 \mathrm{H}_{2} \mathrm{O} \rightarrow \mathrm{N}_{2}+3 \mathrm{C} * \mathrm{O}+6 \mathrm{H}^{+}+6 \mathrm{Cl}^{-}
$$

where $\mathrm{C}^{*}$ and $\mathrm{C}^{*} \mathrm{O}$ have the same meaning as in $\mathrm{Eq}(4)$. Alternatively, trichloramine may be firstly reduced to dichloramine (Eq. (6)), and then dichloramine is reductively decomposed to nitrogen gas (Eq. (4)).

$$
\mathrm{NCl}_{3}+\mathrm{C}^{*}+\mathrm{H}_{2} \mathrm{O} \rightarrow \mathrm{NHCl}_{2}+\mathrm{C}^{*} \mathrm{O}+\mathrm{H}^{+}+\mathrm{Cl}^{-}
$$

\subsection{Effect of water temperature on trichloramine removal}

Figure 3 shows the change in the trichloramine residual ratio with time during contact with SPAC (wood-1). The trichloramine concentration did not decrease with time in the control experiments without PAC dosing (black diamonds), the implication being that the float lid caused the vaporization of trichloramine to be negligibly small in our experimental setup. In contrast, the trichloramine residual ratio decreased with time after the addition of SPAC and declined to approximately 0.05 within $60 \mathrm{~min}$ at $20{ }^{\circ} \mathrm{C}$ (black circles in Fig. 3). Snoeyink and his research group have reported that the time required for sufficient removal of dichloramine by addition of PAC with diameters of 149-177 $\mu$ m was 45 h (Bauer and Snoeyink 1973, Snoeyink and Suidan 1975). In contrast to their results, the time required for trichloramine removal with SPAC in the present study was quite short, most likely a consequence of the increase of the surface area due to the pulverization of commercially available PAC into very 
fine particles.

When the water temperature decreased to $5{ }^{\circ} \mathrm{C}$, the trichloramine residual ratio increased to approximately 0.3 after $60 \mathrm{~min}$ of contact time (white circles in Fig. 3). When the water temperature was decreased to $1{ }^{\circ} \mathrm{C}$, the residual ratio was increased even further (gray circles in Fig. 3). Trichloramine removal with PAC was therefore suppressed at low water temperatures.
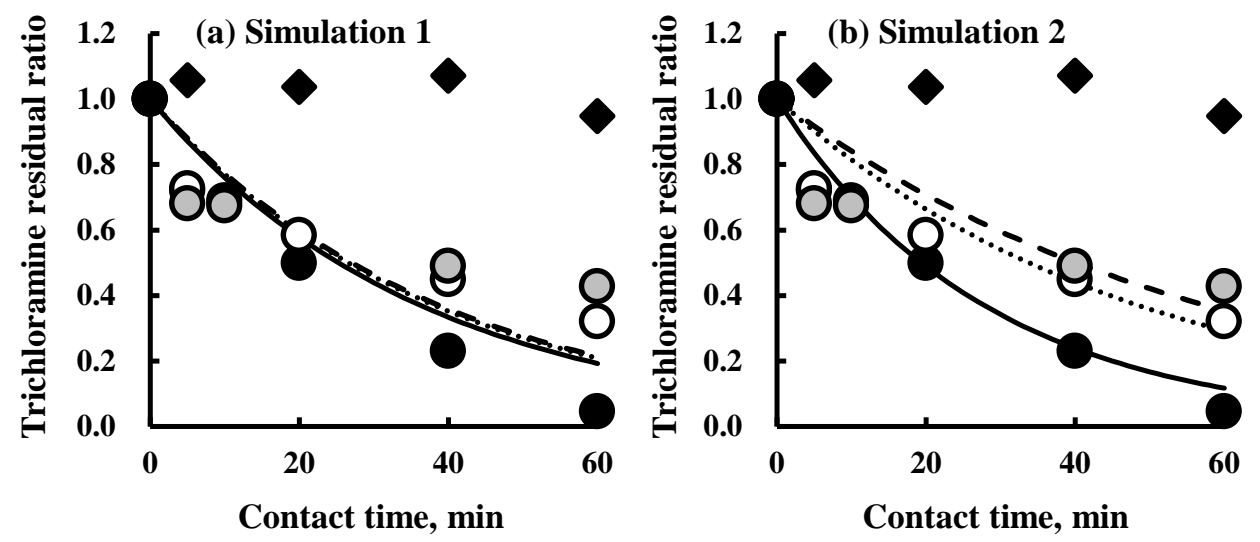

Fig. 3 Trichloramine decay curves with SPAC (wood-1) at different temperatures. Circles and continuous lines represent observed and calculated values, respectively. The SPAC dose was $1 \mathrm{mg} / \mathrm{L} .1^{\circ} \mathrm{C}$, gray circles and dashed line; $5{ }^{\circ} \mathrm{C}$, white circles and dotted line; $20^{\circ} \mathrm{C}$, black circles and solid line; control at $20^{\circ} \mathrm{C}$, black diamonds. (a) Simulation 1: identical values for the trichloramine decomposition rate constant $\left(k_{\mathrm{NCl} 3}\right)$ were used in fitting the calculated values to the observed values. (b) Simulation 2: the $k_{\mathrm{NCl} 3}$ values were determined separately for each temperature by least squares.

One possible explanation for the increase in the trichloramine residual ratio is the decrease of the intraparticle mass transfer rate of trichloramine molecules with decreasing water temperature. The pore diffusion coefficient of trichloramine ( $D_{\mathrm{P}}$, with dimensions of $\mathrm{cm}^{2} / \mathrm{s}$ ) can be expressed as a function of its diffusion coefficient in water as follows:

$$
D_{\mathrm{P}}=\frac{\varepsilon}{\chi} D_{\mathrm{A}}
$$

where $\varepsilon$ and $\chi$ are the porosity and tortuosity (both dimensionless) of the carbon, respectively, and $D_{\mathrm{A}}$ is the diffusion coefficient of trichloramine in water. $D_{\mathrm{A}}$ can be determined with the following equation (Hayduk and Laudie 1974):

$$
D_{\mathrm{A}}=\frac{13.26 \times 10^{-5}}{\eta_{\mathrm{W}}^{1.14} V_{\mathrm{B}}^{\prime 0.589}}
$$

where $\eta_{\mathrm{W}}$ is the viscosity of water (cp), and $V_{\mathrm{B}}$ is the LeBas molar volume of trichloramine $\left(\mathrm{cm}^{3} / \mathrm{mol}\right)$. The pore diffusion coefficient is therefore a function of the viscosity of water, which depends on the temperature of the water. The pore diffusion coefficient therefore depends on the water temperature. The $D_{\mathrm{P}}$ values were calculated to be 1.18 $\times 10^{-6}, 7.37 \times 10^{-7}$, and $6.37 \times 10^{-7} \mathrm{~cm}^{2} / \mathrm{s}$ at temperatures of 20,5 , and $1{ }^{\circ} \mathrm{C}$. The fact that the $D_{\mathrm{P}}$ value decreased 
by about a factor of 2 when the water temperature decreased from $20^{\circ} \mathrm{C}$ to $1{ }^{\circ} \mathrm{C}$ could have an impact on the removal of trichloramine with PAC.

To elucidate the effect of the pore diffusion coefficient on trichloramine removal, simulations were conducted by using the diffusion-reaction model (Eqs. (1-3)). In the simulation, the surface decomposition rate constant of trichloramine $\left(k_{\mathrm{NCl} 3}\right)$, the unknown model parameter, was estimated by determining the value that produced the best fit to the experimental data. In the first simulation (Simulation 1), the value of $k_{\mathrm{NCl}}$ was assumed to be independent of water temperature. As a result, the calculated trichloramine decay curves were almost the same for the three different water temperatures and did not provide a good description of the experimental data (Fig. 3a). The model simulation clearly showed that the differences in diffusive mass transfer rates associated with the differences in water temperatures did not contribute to the differences in trichloramine removal. Therefore, the rate of pore diffusion did not limit the rate of trichloramine removal with SPAC.

Another possible temperature effect would be a temperature-dependent change in the rate of reduction of trichloramine. Chemical reactions are well known to be sensitive to temperature. In the second simulation (Simulation 2), the $k_{\mathrm{NCl} 3}$ values were determined separately by curve fitting at each water temperature. The simulation successfully characterized the observed trichloramine decay curves (Fig. 3b). The $k_{\mathrm{NCl3}}$ values that gave the best fits were $6.5 \times 10^{-5}, 2.8 \times 10^{-5}$, and $2.3 \times 10^{-5} \mathrm{~cm} / \mathrm{s}$ at 20,5 , and $1{ }^{\circ} \mathrm{C}$, respectively. Hence the surface decomposition rate constant of trichloramine decreased with a decrease in water temperature. The increase of the trichloramine residual ratio with decreasing water temperature was therefore attributable to the temperature dependence of the rate constant for the reductive reaction of trichloramine with PAC rather than to a temperature dependence of the diffusive mass transfer rate. The temperature dependence of a reaction rate constant is usually expressed by the Arrhenius equation (Qiang et al. 2014, Song et al. 2014, von Rohr et al. 2014). The fact that the $k_{\mathrm{NCl} 3}$ values determined in the present study at three different water temperatures were well described by the equation $\left(r^{2}=0.998\right)$ (data not shown) supports the validity of our calculation.

Biological treatment, the conventional method for the control of ammonium nitrogen, the major trichloramine precursor, is not expected to produce satisfactory results at low temperatures unless the water is warmed to a temperature better suited for microbiologically mediated nitrification. In contrast, SPAC treatment was found to be only slightly less effective in removing trichloramine at low water temperatures. At low temperatures, SPAC treatment still resulted in the decomposition of trichloramine. Accordingly, an increase in the SPAC dose could result in adequate removal of trichloramine even at low water temperatures. Modifying the SPAC dose based on the water temperature would therefore be very important for effective removal of trichloramine from drinking water at treatment plants and might sometimes require intervention by on-site engineers. However, SPAC treatment is a method for removing trichloramine from finished water that could be superior to traditional biological treatment in terms of the countermeasures required at low water temperatures.

Figure 4 shows the changes in the residual ratio of free chlorine with SPAC contact time. The free chlorine concentration decreased slightly with time, in agreement with the results reported in a previous paper (Snoeyink and Suidan 1972). However, the extent of decrease was quite small compared with the decrease of trichloramine at any water temperature tested. This result means that SPAC treatment could decompose trichloramine selectively, even when trichloramine and free chlorine are present simultaneously in the water. The fact that the strong 
disinfection capability of residual free chlorine could be retained even after effective removal of trichloramine by SPAC treatment is of great relevance to the treatment process.

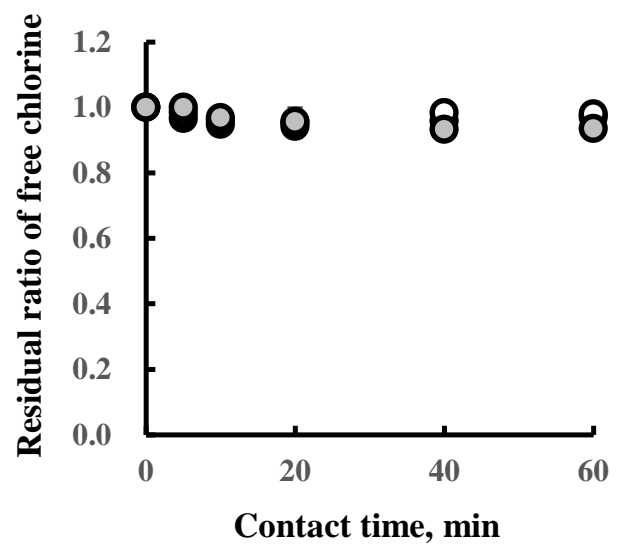

Fig. 4 Residual ratios of free chlorine as a function of time at different water temperatures (wood-1). Gray, white, and black circles indicate results at 1,5 and $20{ }^{\circ} \mathrm{C}$, respectively.

\subsection{Effects of carbon characteristics on trichloramine removal}

Trichloramine decomposition experiments were conducted by using seven different SPACs at $20{ }^{\circ} \mathrm{C}$, and the same methodology used in Simulation 2 was carried out to analyze the experimental results and to obtain the surface decomposition rate constants of trichloramine by the SPACs. Figure 5 shows the surface decomposition rate constants of trichloramine in the presence of the SPACs that we tested. The surface decomposition rate constants of trichloramine tended to be larger for the wood-based SPACs than for the coconut-shell-based and the coal-based SPACs. However, the surface decomposition rate constants of the wood-1 and -4 SPACs were similar to the rate constant of the coconut-1 SPAC. Thus, the differences in surface decomposition rates were not simply explained by the differences in the raw materials used to make the SPACs. To explain the differences in the surface decomposition rates, the physicochemical properties of the SPACs were analyzed and then compared with the surface decomposition rate constants. 


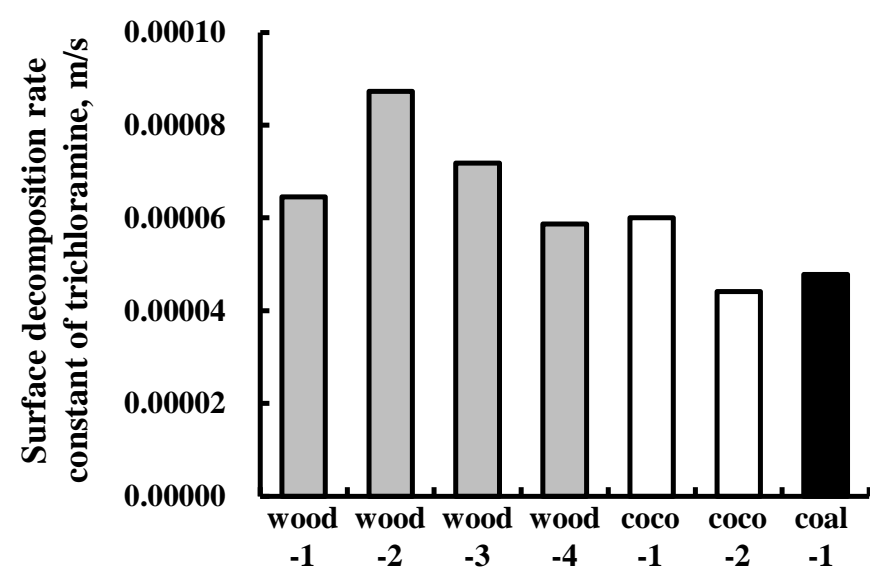

Fig. 5 Trichloramine decomposition rate constants for the SPACs tested in this study.

Table 2 summarizes the correlation coefficients between the trichloramine surface decomposition rate constants and the characteristics of the carbon used to make the SPACs. A weak positive correlation was observed between the surface decomposition rate constant and the equivalent concentration of the basic functional groups $\left(r^{2}\right.$ $=0.37$ ), whereas a moderate negative relationship was observed between the rate constant and the equivalent concentration of the acidic functional groups $\left(r^{2}=0.46\right)$. Although the correlations were not so strong, the fact that the positive-correlation was observed for the basic-functional groups suggests that trichloramine was likely decomposed with the basic functional groups on the surface of the SPACs. Siddiqui et al. (1996) have reported that bromated compounds are reductively decomposed with PAC, and that the removal of bromated compounds is highly correlated with the number of basic functional groups on the surface of a PAC, a result consistent with our results for trichloramine. The surface properties of activated carbon are influenced to a large extent by the foreign elements fixed on the surface, which affect the behavior of the carbon in practical applications (Boehm 1994). The acidic functional groups on activated carbons have been well characterized. Representative examples include carbonylic, carboxylic, phenolic, hydroxylic, lactonic and, quinonic groups (Boehm 1994, 2002). In contrast, the basic functional groups have not been well characterized, and their identity is still being investigated. A pyrone-type structure has been proposed as one of the possible representatives of the basic functional groups (Boehm 2002). Sulfur is easily affixed to the surface of carbon during manufacturing of activated carbons (Donnet 1968), and sulfhydryl groups (-SH) and disulfide (-S-S-) groups have been reported to play a role in the reduction of bromated compounds on the activated carbon surface (Siddiqui et al. 1996). Trichloramine may be reduced by the sulfhydryl group located on the surface of the activated carbon particles, because the sulfhydryl group is known to act as a soft base (Ho 1975) as well as a reductant. The fact that hypochlorous acid (Pereira et al. 1973) and monochloramine (Jacangelo et al. 1987) have been reported to easily react with and to be oxidized by the sulfhydryl group in cysteine supports our hypothesis. To test the hypothesis, the sulfur content of the activated carbon was measured with an elemental analyzer, but no correlation was observed between the surface decomposition rate constant and the sulfur content $\left(r^{2}=0.13\right)$. Whereas the Boehm titration method employed for the quantification of the number of functional groups in the present study determined the concentration of functional groups on the surface of the pore walls of the activated carbon, elemental analysis using the elemental 
analyzer quantified the total number of sulfur atoms, not only on the surface but also within the interior of the activated carbon particles. The fact that no relationship was observed between trichloramine removal and sulfur content may be attributable to the difference between the concentration of functional groups on the surface and within the interior of the activated carbon particles. Elemental analysis may not directly reflect the characteristics of the surface of the pore walls, which must play an important role in the reduction reaction mediated by the activated carbon. Alternatively, the surface of the activated carbon may also have sulfur-containing functional groups in addition to the sulfhydryl group, but some of them may not play a role in the reduction of chloramine. Further study is needed of the sites of the reduction reaction on the carbon surface. Additionally, there were of course a finite number of reduction reaction sites on the carbon surface. The reduction reaction sites may be exhausted when a large amount of trichloramine and/or oxidizing agents is applied relative to the carbon dose. In the future, the consumption of reduction reaction sites on the carbon surface may therefore need to be taken into consideration in the diffusion-reaction model for more accurate prediction of trichloramine removal with activated carbon. In other words, the first-order reaction kinetics that were assumed as a first step in the present study may need to be modified.

Table 2. Coefficients of determination between surface decomposition rate constant of trichloramine and SPAC characteristics

\begin{tabular}{|lr||cc|}
\hline \multicolumn{2}{|c||}{ Carbon characteristics } & sign of correlation & $r^{2}$ \\
\hline \hline Functional group & Basic & + & 0.37 \\
$\left(\mu \mathrm{eeq} / \mathrm{cm}^{2}\right)$ & Acidic & - & 0.46 \\
\hline Elemental composition & $\mathrm{C}$ & - & 0.00 \\
$(\%)$ & $\mathrm{N}$ & - & 0.31 \\
& $\mathrm{~S}$ & - & 0.13 \\
& $\mathrm{O}$ & + & 0.03 \\
\hline ZP $(\mathrm{mV})$ & & - & 0.15 \\
\hline IEP & & - & 0.01 \\
\hline pHpzc & & + & 0.56 \\
\hline
\end{tabular}

$\mathrm{ZP}$, zeta potential; IEP, isoelectric point; $\mathrm{pHpzc}$, $\mathrm{pH}$ at point of zero charge.

A moderate positive correlation was observed between the surface decomposition rate constant and $\mathrm{pH}_{\mathrm{pzc}}\left(r^{2}=\right.$ 0.56): the higher the $\mathrm{pH}_{\mathrm{pzc}}$ of the activated carbon, the larger the surface decomposition rate constant. The $\mathrm{pH}_{\mathrm{pzc}}$ value has been reported to become smaller when the surface of the activated carbon becomes oxidized (Faria et al. 2004, Noh and Schwarz 1990). Accordingly, activated carbon particles whose surfaces are less oxidized are likely to possess a greater ability to remove trichloramine. This observation is consistent with our finding that trichloramine was reductively decomposed to nitrogen gas by contact with activated carbon particles.

\section{Conclusions}

1. Stoichiometric analysis with ${ }^{15} \mathrm{~N}$-labelled trichloramine clearly revealed that trichloramine was reductively 
transformed into nitrogen gas by contact with PAC.

2. The weak positive correlation observed between the surface decomposition rate constant of trichloramine and the concentration of basic functional groups suggested that trichloramine was reduced by the sulfhydryl groups (-SH) located on the surface of the activated carbon particles.

3. SPACs with a median diameter of $\approx 0.5 \mu \mathrm{m}$ quite effectively decomposed trichloramine; approximately 300 $\mu \mathrm{g}-\mathrm{Cl}_{2} / \mathrm{L}$ of trichloramine was decomposed to a residual ratio of $<0.05$ within $60 \mathrm{~min}$ by addition of $1 \mathrm{mg} / \mathrm{L}$ of SPAC.

4. The residual ratio of trichloramine after SPAC dosing increased at low water temperatures $\left(1-5^{\circ} \mathrm{C}\right)$, but the SPAC still decomposed trichloramine at a rate that was practical for water treatment. Theoretical analysis with the diffusion-reaction model revealed that the increase in the trichloramine residual ratio with decreasing water temperature was attributable to the temperature dependency of the reaction rate constant rather than to the temperature dependence of the rate of diffusive mass transfer.

5. SPAC could decompose trichloramine selectively, even when trichloramine and free chlorine were present simultaneously in the water. The fact that free chlorine was still present, even after trichloramine was effectively decomposed by the SPAC, indicated that the strong disinfection capability of free chlorine was retained even after the SPAC treatment.

\section{Acknowledgements}

This research was supported in part by a Grant-in-Aid for Scientific Research S (No. 24226012) from the Japan Society for the Promotion of Science, by a Health and Labour Sciences Research Grant (Research on Health Security Control) from the Ministry of Health, Labor and Welfare of Japan, and by the Bureau of Waterworks, Tokyo Metropolitan Government.

\section{References}

American Public Health Association/American Water Works Association/Water Environment Federation (2005) Standard Methods for Examination of Water and Wastewater, American Public Health Association.

Andersson, A., Laurent, P., Kihn, A., Prévost, M. and Servais, P. (2001) Impact of temperature on nitrification in biological activated carbon (BAC) filters used for drinking water treatment. Water Res 35, 2923-2934.

Bao, M., Griffini, O., Santianni, D., Barbieri, K., Burrini, D. and Pantani, F. (1999) Removal of bromate ion from water using granular activated carbon. Water Res 33, 2959-2970.

Bauer, R.C. and Snoeyink, V.L. (1973) Reactions of chloramines with active carbon. Journal Water Pollution Control Federation 45, 2290-2301.

Boehm, H.P. (1994) Some aspects of the surface chemistry of carbon blacks and other carbons. Carbon 32, 759-769.

Boehm, H.P. (2002) Surface oxides on carbon and their analysis: a critical assessment. Carbon 40, 145-149.

Çapar, G.e. and Yetış, Ü. (2002) Removal of THM precursors by GAC: Ankara case study. Water Res 36, 
1379-1384.

Donnet, J.B. (1968) The chemical reactivity of carbons. Carbon 6, 161-176.

Faria, P.C.C., Órfão, J.J.M. and Pereira, M.F.R. (2004) Adsorption of anionic and cationic dyes on activated carbons with different surface chemistries. Water Res 38, 2043-2052.

Giles, M.A. and Danell, R. (1983) Water dechlorination by activated carbon, ultraviolet radiation and sodium sulphite: A comparison of treatment systems suitable for fish culture. Water Res 17, 667-676.

Gonce, N. and Voudrias, E.A. (1994) Removal of chlorite and chlorate ions from water using granular activated carbon. Water Res 28, 1059-1069.

Hayduk, W. and Laudie, H. (1974) Prediction of diffusion coefficients for non-electrolysis in dilute aqueous solutions. AIChE Journal 20, 611-615.

Herzing, D.R., Snoeying, V.L. and Wood, N.F. (1977) Activated carbon adsorption of the odorous compounds 2-methylisoborneol and geosmin. Journal of American Water Works Association 69, 223-228.

Ho, T.-L. (1975) Hard soft acids bases (HSAB) principle and organic chemistry. Chemical Reviews 75, 1-20.

Jacangelo, J.G., Olivieri, V.P. and Kawata, K. (1987) Oxidation of sulfhydryl groups by monochloramine. Water Res 21, 1339-1344.

Kosaka, K., Seki, K., Kimura, N., Kobayashi, Y. and Asami, M. (2010) Determination of trichloramine in drinking water using headspace gas chromatography/mass spectrometry. Water Science and Technology: Water Supply 10, 23-29.

Kranser, S.W. and Barret, S.E. (1984) Aroma and flavor characteristic of free chlorine and chloramines. Proceedings of AWWA WQTC.

Matsui, Y., Aizawa, T., Kanda, F., Nigorikawa, N., Mima, S. and Kawase, Y. (2007) Adsorptive removal of geosmin by ceramic membrane filtration with super-powdered activated carbon. Journal of Water Supply: Research and Technolog-AQUA 56, 411-418.

Matsui, Y., Ando, N., Sasaki, H., Matsushita, T. and Ohno, K. (2009) Branched pore kinetic model analysis of geosmin adsorption on super-powdered activated carbon. Water Res 43, 3095-3103.

Matsui, Y., Murai, K., Sasaki, H., Ohno, K. and Matsushita, T. (2008) Submicron-sized activated carbon particles for the rapid removal of chlorinous and earthy-musty compounds. Journal of Water Supply: Research and Technology-AQUA 57, 577-583.

Matsui, Y., Murase, R., Sanogawa, T., Aoki, N., Mima, S., Inoue, T. and Matsushita, T. (2005) Rapid adsorption pretreatment with submicron powdered activated carbon particles before microfiltration. Water Science and Technology 51, 249-256.

Noh, J.S. and Schwarz, J.A. (1990) Effect of HNO3 treatment on the surface acidity of activated carbons. Carbon 28, 675-682.

Pereira, W.E., Hoyano, Y., Summons, R.E., Bacon, V.A. and Duffield, A.M. (1973) Chlorination studies II. The reaction of aqueous hypochlorous acid with $\alpha$-amino acids and dipeptides. Biochimica et Biophysica Acta (BBA) - General Subjects 313, 170-180.

Piriou, P., Mackey, E.D., Suffet, I.H. and Bruchet, A. (2004) Chlorinous flavor perception in drinking water. Water Science and Technology 49, 321-328. 
Qiang, Z., Tian, F., Liu, W. and Liu, C. (2014) Degradation of methiocarb by monochloramine in water treatment: Kinetics and pathways. Water Res 50, 237-244.

Robeck, G.G., Dostal, K.A., Cohen, J.M. and Kreissl, J.F. (1965) Effectiveness of water treatment processes in pesticide removal. Journal of American Water Works Association 57, 181-199.

Siddiqui, M., Zhai, W., Amy, G. and Mysore, C. (1996) Bromate ion removal by activated carbon. Water Res 30, 1651-1660.

Snoeyink, V.L. and Suidan, M.T. (1972) Disinfect -Water and Wastewater, Ann Arbor Science Publishers Inc., MI, USA.

Snoeyink, V.L. and Suidan, M.T. (1975) Water and Wastewater, Ann Arbor Science.

Song, D., Liu, H., Qiang, Z. and Qu, J. (2014) Determination of rapid chlorination rate constants by a stopped-flow spectrophotometric competition kinetics method. Water Res 55, 126-132.

Sontheimer, H., Crittenden, J.C. and Summers, R.S. (1988) Activated carbon for water treatment, 2nd ed., DVGW-Forschungsstelle, Karlsruhe, Germany.

Štembal, T., Markić, M., Ribičić, N., Briški, F. and Sipos, L. (2005) Removal of ammonia, iron and manganese from groundwaters of northern Croatia—pilot plant studies. Process Biochemistry 40, 327-335.

Suidan, M.T., Snoeyink, V.L. and Schmitz, R.A. (1977) Reduction of aqueous free chlorine with granular activated carbon - pH and temperature effects. Environmental Science \& Technology 11, 785-789.

von Rohr, R.M., Hering, J.G., Kohler, H.-P.E. and von Gunten, U. (2014) Column studies to assess the effects of climate variables on redox processes during riverbank filtration. Water Res 61, 263-275.

Voudrias, E.A., Dielmann Iii, L.M.J., Snoeyink, V.L., Larson, R.A., McCreary, J.J. and Chen, A.S.C. (1983) Reactions of chlorite with activated carbon and with vanillic acid and indan adsorbed on activated carbon. Water Res 17, 1107-1114. 